\title{
Manejo anestésico del paciente quemado
}

\author{
Anesthetic management of the burn patient \\ Dr. Miguel Ángel García-Lara,* Dra. Alejandra Sánchez-Sagrero, ${ }^{\ddagger}$ \\ Dr. Alejandro Aguilar-Águila-Martínez, ${ }^{\S}$ Dra. Nora Lorena López-León
}

Citar como: García-Lara MÁ, Sánchez-Sagrero A, Aguilar-Águila-Martínez A, López-León NL. Manejo anestésico del paciente quemado. Rev Mex Anestesiol. 2022; 45 (2): 129-134. https://dx.doi.org/10.35366/103888

RESUMEN. El conocimiento de la fisiopatología de las quemaduras son base del manejo perioperatorio. Todos los órganos y sistemas resultan alterados y los cambios hemodinámicos en la fase aguda se caracterizan por una disminución del gasto cardíaco. Después de seis días de la lesión, ocurre un nuevo cambio hemodinámico, esta fase es conocida como estado hipermetabólico y su duración está en relación con la extensión y profundidad de la lesión. La lesión por inhalación es un factor importante a considerar como mal pronóstico y, de igual manera, el número de comorbilidades y/o trauma asociado. Durante este segundo período, los anestesiólogos tienen una participación continua y estrecha. Los problemas farmacológicos, manejo de vía aérea, accesos vasculares, hipotermia, monitoreo y control del dolor, entre otros, se hacen muy marcados. Por último, la fase de secuelas acompaña al paciente para toda su vida y la magnitud de los daños, costos y discapacidad serán directamente relacionados con la atención administrada durante las primeras dos fases.

ABSTRACT. Knowledge of the pathophysiology of burns is the basis of perioperative management. All organs and systems are altered and hemodynamic changes in the acute phase are characterized by a decrease in cardiac output. After six days of injury a new hemodynamic change occurs, this phase is known as hypermetabolic state and its duration is in relation to the extent and depth of the injury. Inhalation injury is an important factor to consider as a poor prognosis and also the number of associated comorbidities and/or trauma. During this second period the Anesthesiologists have a continuous and close participation. Pharmacological problems, airway management, vascular access, hypothermia, monitoring and pain control among others become very marked. Finally, the phase of sequelae is for life and the magnitude of damages, costs and disability will be directly related to the care administered during the first two phases.

\section{MANEJO ANESTÉSICO DEL PACIENTE CON QUEMADURAS}

$\mathrm{E}$ l Sistema Nacional de Vigilancia Epidemiológica reportó que para el 2013 se presentó un total de 126,786 nuevos casos de quemaduras en México, siendo el Instituto Mexicano del Seguro Social el que atendió el 65.5\%, Secretaría de Salud el 21.4\% y el resto se lo repartió el ISSSTE, PEMEX y otras instituciones. Los grupos más afectados son de 25 a 44 años con 43,000 casos aproximadamente, seguido de los niños de uno a cuatro años con 13,864 casos. La mayoría de las quemaduras son accidentes prevenibles con un alto grado de mortalidad, costos y discapacidad ${ }^{(1)}$.

El objetivo de este trabajo es poner a consideración de los anestesiólogos interesados los conceptos actuales relacionados con la fisiopatología y manejo perioperatorio del paciente quemado, con la finalidad de obtener el mejor resultado y el menor número de secuelas.

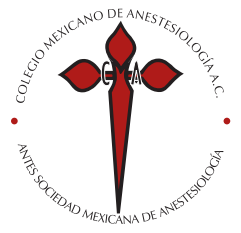

Palabras clave:

Quemaduras, anestesia, vía aérea en quemados, valoración preoperatoria del quemado, manejo de líquidos en quemaduras, monitoreo en quemaduras.

Keywords:

Burns, burn anesthesia, burned airway, preoperative burn assessment, burn fluid management, burn monitoring.

* Jefe de Anestesiología del Centro Nacional de Investigación y Atención de Quemados. Instituto Nacional de Rehabilitación «Dr. Luis Guillermo Ibarra Ibarra». ‡ Residente de segundo año de Anestesiología Pediátrica, Instituto Nacional de Pediatría. § Residente de segundo año de Anestesiología. Centro de Alta Especialidad «Dr. Rafael Lucio». " Adscrita de Anestesiología, Hospital General «Dr. Manuel Gea González».

Correspondencia:

Dr. Miguel Ángel García-Lara Centro Nacional de Investigación y Atención de Quemados. Instituto Nacional de Rehabilitación «Dr. Luis Guillermo Ibarra Ibarra». E-mail: miguelgarcianest@ gmail.com

Recibido: 28-03-2019 Aceptado: 15-05-2019 


\section{Valoración preoperatoria}

a) Fisiopatología de las quemaduras

La respuesta inflamatoria que se produce ante una quemadura extensa (mayor de 30\% superficie corporal SCQ) produce la exposición continua y por mucho tiempo a mediadores inflamatorios (Tabla 1). El efecto sistémico de esta exposición progresa de la siguiente manera: fase de choque por quemaduras (fase aguda o Ebb), que corresponde a las primeras 48 a 72 horas $^{(2)}$. La fase hipermetabólica (fase Flow), que inicia en la primera semana y puede durar varios años, dependiendo de la extensión y complicaciones ${ }^{(3)}$. Por último, esta fase de secuelas de una quemadura acompañan al paciente para toda su vida. Es por eso que durante la valoración preoperatoria debemos enfocarnos en la fase de evolución, interrogatorio y exploración para determinar qué otras enfermedades o traumas acompañan al paciente ${ }^{(4)}$.

\section{b) Lesión por inhalación}

La lesión por inhalación representa un aumento de la probabilidad de muerte de más del 30\%. El estudio diagnóstico por excelencia es la broncoscopía, que evidenciará diferentes grados de daño (Tabla 2$)^{(5)}$. Por otro lado, el número de comorbilidades tiene una asociación con la estancia hospitalaria y sobrevida (Tabla 3)(6).

\section{c) Evaluación clínica}

El choque por quemaduras es mixto (hipovolémico, distributivo y cardiogénico). Lo esperado en la valoración durante la fase aguda sería encontrar paciente con bajo gasto cardíaco, hematocrito elevado, oliguria, acidosis metabólica, disminución de la saturación venosa de oxígeno, síndrome compartimental, mioglobulinuria, alteración del estado de alerta, falla respiratoria y renal ${ }^{(4)}$.

Durante la fase hipermetabólica, el paciente presentará aumento del gasto cardíaco, frecuencia cardíaca y presión arterial, también observamos aumento de la gluconeogenia, hígado graso, alteración del metabolismo hepático, disminución de la hematopoyesis, tiempos de coagulación prolongados, anemia, inmunosupresión, edema pulmonar, neumonía, falla renal, catabolismo muscular acelerado, hipoalbuminemia, desnutrición, resistencia a la insulina, sepsis, falla orgánica múltiple ${ }^{(4)}$. La valoración de esta etapa debe enfocarse en los posibles tratamientos administrados para mitigar la respuesta hipermetabólica: beta-bloqueador, infusión de insulina, hipoglucemiantes orales, uso de anabólicos, alfa 2-agonistas, antibióticos, analgésicos, etcétera ${ }^{(7,8)}$. Así también, los procedimientos quirúrgicos son frecuentes con alto grado de sangrado, por lo que debe tomar precauciones solicitando un número adecuado de derivados sanguíneos, basado en un cálculo de sangrado esperado (Tabla 4) ${ }^{(9)}$.

\section{Vía aérea (VA) en quemaduras}

Durante una quemadura grave, el abordaje de la VA es prácticamente obligatorio, y la dificultad estará asociada directamente al trauma y en modo secundario al tratamiento y a las complicaciones asociadas al abordaje continuo; es decir, que los pacientes gran quemado pasarán por vía aérea difícil (VAD) en algún momento del largo tratamiento ${ }^{(9,10)}$.

\section{a) Valoración de vía aérea}

El trauma directo en cabeza, las quemaduras en cara o vía aérea, lesión por inhalación, el sangrado, vómito, exudado, inestabilidad hemodinámica y el edema asociado a la resucitación de líquidos son los primeros factores por los que se debe realizar una intubación ${ }^{(4)}$. El término intubación profiláctica ${ }^{(11)}$ está diseñado para aquellos pacientes con riesgo de edema, en donde lo que se busca es colocar el tubo endotraqueal del tamaño y características más adecuadas antes de que el calibre de la VA disminuya. Los factores para predecir probabilidad de edema son: a) quemaduras mayores del 50\% SCQ, b) quemadura en cara, cuello, tórax y abdomen circunferencial, c) necesidad de altas dosis de opioide, d) traslados de larga distancia a unidad de quemados y e) dificultad para la deglución o respiración ${ }^{(12)}$. Actualmente, existe evidencia de que sólo utilizar indiscriminadamente los criterios clínicos anteriores lleva a intubación innecesaria de más de un tercio de todos los pacientes que actualmente se les coloca un tubo endotraqueal $^{(13)}$. Se sugiere actuar más prudentemente e iniciar con un apoyo con oxígeno por mascarilla facial con
Mediador

Histamina, prostaciclina, prostaglandinas, tromboxanos bradicinina, serotonina Leucotrienos

Catecolaminas

Angiotensina, vasopresina y factor agregante de plaquetas
Efecto central y tisular

Hipotensión, aumento de permeabilidad, vasodilatación

Hipertensión pulmonar

Disfunción cardíaca y daño tisular

Elevación de presión, isquemia gastrointestinal y vasoconstricción 


\begin{tabular}{|c|c|c|}
\hline \multicolumn{3}{|c|}{$\begin{array}{l}\text { Tabla 2: Grados de lesión por inhalación por } \\
\text { broncoscopía. }\end{array}$} \\
\hline Grado & Clasificación & Daño \\
\hline 0 & No hay lesión & Sin alteraciones \\
\hline 1 & Lesión leve & Eritema \\
\hline 2 & Lesión moderada & $\begin{array}{l}\text { Eritema, broncorrea, depósitos } \\
\text { carbonáceos }\end{array}$ \\
\hline 3 & Lesión severa & Broncorrea, úlceras \\
\hline 4 & Daño masivo & Necrosis \\
\hline
\end{tabular}

Tabla 3: Relación entre número de comorbilidades y resultado.

Características

Días de estancia en UCl Muerte (\%)

$\begin{array}{rrrc}0 & 1 & 2 & 3 \text { o más } \\ 11 & 16 & 18 & 22 \\ 5 & 9 & 13 & 20\end{array}$

reservorio y sólo realizar la intubación al primer dato de dificultad respiratoria en aquellos pacientes con los factores de riesgo y/o inestabilidad hemodinámica ${ }^{(13)}$.

\section{b) Manejo de vía aérea}

La intubación repetida de la vía aérea para todos los eventos quirúrgicos aumenta el riesgo de desarrollar estenosis traqueal $^{(4)}$. El vaciamiento gástrico está disminuido en los pacientes con lesión extensa; se consideran estómago lleno. La VAD está asociada a ventilación difícil por todos los factores relacionados a no poder hacer sellado de la mascarilla facial (Figura 1) como son: quemaduras, exudado, apósitos, vendajes, cremas, sondas, sangrado, deformidad, etcétera $^{(11)}$. Con el paso del tiempo, las contracturas hacen aparición y la extensión del cuello, subluxación mandibular y la apertura oral generan el punto crítico de la VAD; la intubación difícil tiene mayor relación con las contracturas de las heridas. Usar los predictores para diagnosticar VAD puede generar error, ya que la VA se encuentra distorsionada. Use los criterios diagnósticos con precaución y ponga énfasis en la historia clínica, enfóquese en las intubaciones más recientes para predecir el grado de dificultad con base en complicaciones previas o posible mejoría en el manejo $^{(4,11)}$.

Recuerde que los dispositivos supraglóticos de rescate pueden ser difíciles de colocar, principalmente cuando la apertura oral es muy limitada. Siempre que sea necesaria la intubación de la VA de un gran quemado, pida ayuda de inicio y cuente con diversas habilidades para realizar intubación (despierto, retrógrada, etc.) así como de varios equipos para resolver las dificultades (cánulas de Guedel, laringoscopios con varios tipos de hojas, guía metálica, videolaringoscopio, fibroscopio) ${ }^{(4,9)}$.

\section{Cambios farmacológicos en el paciente quemado}

\section{a) Farmacocinética y farmacodinamia}

Después de una lesión por quemadura, la farmacocinética y farmacodinamia serán diferentes a las de los pacientes no quemados. Existen alteraciones en compartimentos, cambios en el gasto cardíaco, variabilidad en la perfusión de los órganos, alteración de función renal y hepática, cambios en los niveles de proteínas séricas, tolerancia y nuevas vías de eliminación ${ }^{(14)}$.

Las respuestas de los pacientes quemados a los fármacos es irregular. Los pacientes que llegan a la sala de forma aguda después de una quemadura pueden estar en choque hipovolémico con depresión miocárdica, y debe tomar en cuenta estos factores para calcular los fármacos y dosis a administrar; por lo general, deberá disminuir las dosis, ya que existe alto riesgo de empeorar el estado hemodinámico ${ }^{(4)}$.

El estado hipermetabólico es la fase de mayor contacto entre el anestesiólogo y el paciente. Múltiples procedimientos hacen esta fase la más complicada. Hablando de farmacocinética, el volumen de distribución se modifica de una manera importante: aumenta igualmente la vía de eliminación a través de la zona

\begin{tabular}{|c|c|}
\hline \multicolumn{2}{|c|}{$\begin{array}{l}\text { Tabla 4: Pérdidas sanguíneas esperadas } \\
\text { durante la escisión de la quemadura. }\end{array}$} \\
\hline $\begin{array}{l}\text { Día del procedimiento quirúrgico } \\
\text { desde la lesión }\end{array}$ & Sangrado esperado $\left(\mathrm{mL} / \mathrm{cm}^{2}\right)$ \\
\hline Menos de 24 horas & 0.45 \\
\hline 3 días & 0.65 \\
\hline 3 a 15 días & 0.75 \\
\hline Infección o más de 16 días & 1.25 \\
\hline
\end{tabular}

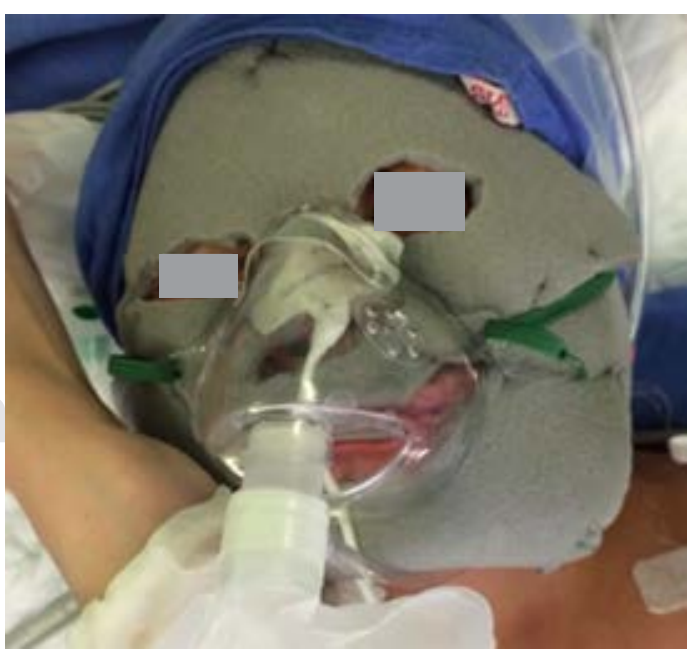

Figura 1: Ventilación difícil por apósitos en cara. 
de trauma; la falla renal y hepática, alteración de la actividad enzimática de fase 1 (oxidación, reducción, desmetilación e hidoxilación) y sangrado generan un segundo punto que altera el cálculo del decaimiento de una concentración plasmática ${ }^{(4,9,14)}$. Estos cambios hacen difícil la tarea de predecir o calcular dosis de carga o mantenimiento de los anestésicos. Por otro lado, la farmacodinamia nos dice que se presenta una marcada hipoalbuminemia y elevación de la alfa 1 glicoproteína ácida, factor relacionado con la fracción libre del fármaco y razón importante que afecta la duración de acción del mismo ${ }^{(4,14)}$.

\section{b) Opioides}

La tolerancia está muy marcada y esto dificulta el manejo del dolor. El manejo transoperatorio obliga a dos medidas, por una parte, al uso de dosis mayores y, por otra, a considerar agregar coadyuvantes como dexmedetomidina o ketamina ${ }^{(4,14)}$.

\section{c) Relajantes musculares}

Existe un franco daño de los receptores acetilcolina de la placa neuromuscular. La presencia de receptores denervados en gran cantidad a partir de las 48 horas de la lesión, con la conformación de cinco subunidades 7 alfa, hacen al receptor muy afín por acetilcolina, alterando el tiempo y flujo del potasio. Estos receptores presentarán una regulación a la alza. La respuesta es una hipersensibilidad a la succinilcolina con alto riesgo de hipercalemia, generando contraindicación absoluta para su uso después del segundo día de quemadura y una baja respuesta a los relajantes no despolarizantes, lo que implica aumentar la dosis entre tres a cinco veces para observar su efecto. Esta alteración puede durar hasta dos años ${ }^{(4,9,14)}$.

\section{d) Ketamina}

Excelente por su acción en receptores NMDA para generar analgesia, debe ser manejada junto con benzodiacepinas para reducir la incidencia de disforia. Los persistentes niveles altos de catecolaminas en los quemados genera una desensibilización y regulación a la baja de los beta adrenorreceptores y, como resultado, un efecto de depresión miocárdica directa ${ }^{(4)}$.

\section{e) Anestésicos inhalados}

Su vía de administración facilita y asegura la dosificación; sin embargo, pueden causar vasodilatación y depresión miocárdica. ${ }^{(9)}$

\section{Monitoreo}

Los valores obtenidos por el monitoreo transoperatorio son básicos para realizar el manejo hemodinámico durante el tratamiento de una quemadura, nos ayudan a tomar adecuadas decisiones para el manejo de líquidos, uso de anestésicos y dosis, así como para la administración de aminas o tratamiento de complicaciones, como el paro cardíaco ${ }^{(4)}$.

La obtención de los valores del monitoreo no es sencilla. La distorsión, las quemaduras, amputación y la necesidad de realizar tratamiento quirúrgico en la zona de colocación de los dispositivos generan la necesidad constante de estar modificando su ubicación ${ }^{(4,9)}$. El trazo eléctrico se ve afectado por la colocación de apósitos con plata, uso de electrocauterio y soluciones, generando interferencia y pérdida de señal. Use grapas, vendas o suturas para fijar aquellos dispositivos que necesitan adherirse (electrocardiograma, BIS, entropía) ${ }^{(4,9)}$.

No olvide revisar temperatura; la hipotermia es uno de los puntos a evitar durante el tratamiento ${ }^{(4,9)}$. Recuerde que todo el monitoreo utilizado se ensucia fácilmente, convirtiéndose en fómite ${ }^{(4)}$.

El monitoreo invasivo es indispensable, principalmente en las quemaduras muy extensas o con inestabilidad hemodinámica. Los valores de lectura obtenidos a través de ellos hacen más fácil la tarea de dirigir los líquidos. Actualmente, uno de los principales orígenes de la sepsis en quemaduras son los accesos vasculares, por lo que deberá colorarlos con base en el riesgo-beneficio (Figura 2)(4,9,15).

\section{Control de temperatura}

La fisiopatología del estado hipermetabólico produce una alteración a nivel hipotalámico, elevando la temperatura central. Las pérdidas por evaporación se incrementan directamente relacionado con la extensión de la lesión; es fácil observar hipotermia durante un procedimiento quirúrgico. Este proceso es mal tolerado, pues aumenta el consumo de oxígeno y exacerba la respuesta catabólica. El proceso de calentamiento debe realizarse por múltiples vías: calentar las soluciones intravenosas, utilizar filtro de humidificador nariz, elevar la temperatura del quirófano $\left(32 \text { a } 38{ }^{\circ} \mathrm{C}\right)^{(4,9)}$, uso de calentadores de radiación, minimizar las zonas de exposición, cubrir cabeza y extremidades con calentadores o sabanas térmicas. Asegurar que todas las compresas y soluciones de infiltración se calienten y cambien constantemente ${ }^{(4)}$.

\section{Accesos vasculares}

Su manejo es complicado y delicado por el trauma y el edema. Debe tomar en cuenta que son fuente de infección, y deberá considerar su reemplazo, así como la zona a colocar. El uso del ultrasonido es una buena guía para colocación en casos difíciles ${ }^{(4,9)}$.

\section{Manejo de líquidos}

Es uno de los retos más importantes durante el perioperatorio. Actualmente, la tendencia más marcada nos dice que el ma- 


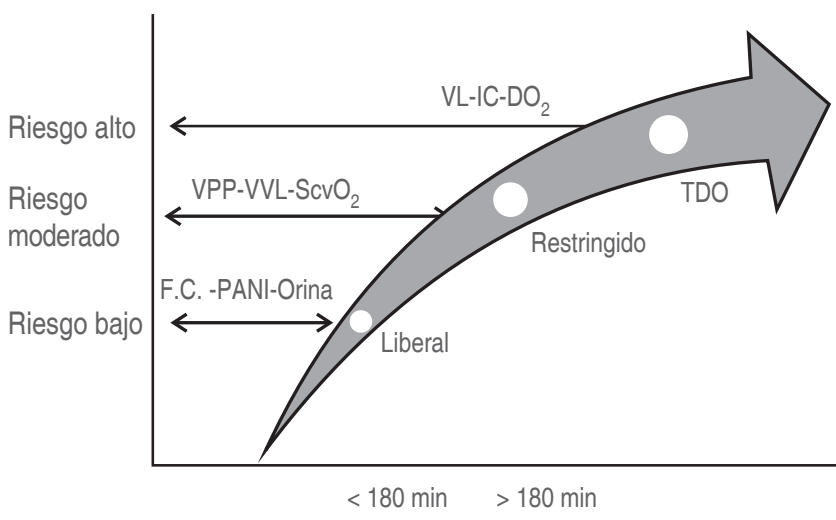

Figura 2: Presión arterial no invasiva.

VPP $=$ Variabilidad pletismográfica, $\mathrm{VVL}=$ Variabilidad del volumen latido $\mathrm{ScvO}_{2}=$ Saturación venosa central, $\mathrm{VL}=$ Volumen latido, IC = Índice cardíaco, $\mathrm{DO}_{2}=$ Deliberación de Oxígeno.

nejo de los líquidos guiado por objetivos individualizado es una de las mejores formas de garantizar la adecuada perfusión tisular (Tabla 5) ${ }^{(9,15)}$. El grado de mediciones dependerá del tiempo quirúrgico y del estado crítico del paciente. Evite el uso de coloides, y, en caso de ser necesario, use albúmina. Durante el transoperatorio existe un alto riesgo de sangrado. Considere el uso de un protocolo de transfusión masiva como opción a la reposición ${ }^{(4)}$. Recuerde que cada paquete puede aumentar el riesgo de muerte hasta en un 13\% y de desarrollar lesión pulmonar asociada a trasfusión, por lo que buscar valores de hemoglobina entre 7 a $9 \mathrm{~g} / \mathrm{dL}$ es considerable y seguro, a menos que el paciente tenga riesgos de seguir sangrando ${ }^{(15)}$.

\section{Técnica anestésica}

La sedación se deja para procedimientos pequeños como cambios de apósitos en pacientes estables. El control del dolor y la disminución del consumo de opioides brindado por la anestesia regional no tienen comparación; sin embargo, las alteraciones en la coagulación, infecciones cerca del sitio de punción y quemaduras extensas limitan su aplicación. Por lo que la vía se centra a dos modos: general balanceada y anestesia total endovenosa (TIVA). Ninguna de las dos manifiesta supe-

\section{Tabla 5: Objetivos del manejo de líquidos.}

Presión arterial normal y gasto urinario de 1 a $2 \mathrm{~mL} / \mathrm{kg} / \mathrm{h}$

Exceso de base $(\leq 5)$ y lactato $2 \mathrm{mmol} / \mathrm{l}$

Índice cardíaco $4.5 \mathrm{l} / \mathrm{min} / \mathrm{m}^{2}$

Variabilidad pletismográfica $<13 \%$

Índice de deliberación de oxígeno $600 \mathrm{~mL} / \mathrm{min} / \mathrm{m}^{2}$

rioridad estadísticamente significativa sobre la otra y deberá individualizarse cada caso para determinar los beneficios que podría obtener el paciente por alguna de ellas; sin embargo, los problemas del acceso vascular y los grandes cambios farmacológicos complican la administración de la TIVA ${ }^{(4)}$.

\section{Cuidados postoperatorios}

El paciente quemado constantemente deberá salir a la Unidad de Terapia Intensiva. La técnica de cirugía de control de daños nos dice que el paciente pasará varias veces por la fase 4 y 5 (cirugía y estabilización en Terapia Intensiva). El control del dolor debe ser multimodal a base de uso de paracetamol, AINE, opioides, ketamina, alfa 2 agonistas, neuromoduladores, siempre y cuando la situación clínica lo permita ${ }^{(4,9)}$.

\section{CONCLUSIÓN}

El manejo multidisciplinario de las quemaduras involucra de forma importante al anestesiólogo. Los pacientes con quemadura requieren varias cirugías. Los cambios fisiopatológicos afectan importantemente el cuidado perioperatorio $^{(3)}$. La anticipación a la presentación de complicaciones es de suma importancia, por lo que el tratamiento temprano con cobertura cutánea lo antes posible siempre será la mejor forma de resolver una quemadura ${ }^{(9)}$. Los cambios farmacológicos, la VAD, los retos de un adecuado manejo de líquidos, grandes pérdidas sanguíneas, riesgo de sepsis e hipotermia son los grandes retos que un anestesiólogo enfrentará ante aquellas personas con gran extensión de quemaduras $^{(4)}$.

\section{REFERENCIAS}

1. Moctezuma PL, Páez FI, Jiménez GS, Miguel HK, Foncerrada OG, Sánchez A y cols. Epidemiología de las quemaduras en México. Rev Esp Méd Quir. 2015;20:78-82.

2. Latenser BA. Critical care of the burn patient: the first 48 hours. Crit Care Med. 2009;37:2819-2826.

3. Jewo PI, Fadeyibi IO. Progress in burns research: a review of advances in burn pathopysiology. Ann Burns Fire Disasters. 2015;28:105-115.

4. Bittner EA, Shank E, Woodson L, Martyn JA. Acute and perioperative care of the burn-injured patient. Anesthesiology. 2015;122:448-464.
5. Spano S, Hanna S, Li Z, Wood D, Cartotto R. Does bronchoscopic evaluation of inhalation injury severity predict outcome? J Burn Care Res. 2016;37:1-11.

6. Thombs BD, Singh VA, Halonen J, Diallo A, Milner SM. The effects of preexisting medical comorbidities on mortality an length of hospital stay and acute burn injury. Ann Surg 2007;245:629-634.

7. Diaz EC, Herdon DN, Porter C, Sidossis LS, Suman OE, Borsheim E. Effects of pharmacological interventions on muscle protein synthesis and breakdown in recovery from burns. Burns. 2015;41:649-657. 
8. Rojas Y, Finnerty CC, Radhakrishnan RS, Herdon DN. Burns: an update on current pharmacotherapy. Expert Opin Pharmacother. 2012;13:2485-2494.

9. Woodson L, Sherwood E, Aarsland A, Talon M, Kinsky MP, Morvant EM. Anesthesia for burned patients. In: Herndon DN. Total burn care. 4th. ed., Edinburgh: Saunders Elsevier, 2012, pp. 173-205.

10. Schmitz B, Griswold J. Airway management in burn patients. In: Hagberg C. Benumof's and Hagberg's airway management. Philadelphia : Elsevier/Saunders, 3rd. ed., 2013, pp. 902-911.

11. García LM. Vía aérea difícil del paciente gran quemado y con secuelas de quemaduras. Rev Mex Anest. 2014;37:S222-S225.
12. Kramer G. Pathopysiology of burn shock and burn edema. In: Herdon D. Total burn care. Edinburgh: Saunders Elsevier, 4th. ed., 2012, pp. 104-117.

13. Romanowski K, Palmieri TI, Sen S, Greenhalgh DG. More than one third of intubations in patients transferred to burn centers are unnecessary: proposed guidelines for appropriate intubation of the burn patient. Burn Care Res. 2016;37:e409-e414.

14. Blanchet B, Jullien V, Vinsonneau C, Tod M. Influence of burns on pharmacokinetics and parmacodynamics of drugs used in the care of burn patients. Clin Pharmacokinet. 2008;47:635-654.

15. García LM. Manejo de líquidos en el perioperatorio del paciente quemado. Rev Mex Anest. 2015;38:S230-S233. 